\title{
CIRUGÍA DEL PTERIGIÓN: ESTUDIO COMPARATIVO ENTRE AUTOINJERTO CONJUNTIVAL CON SUTURA Y CON ADHESIVO TISULAR
}

\author{
PTERYGIUM SURGERY: COMPARATIVE STUDY OF \\ CONJUNCTIVAL AUTOGRAFT WITH SUTURE VERSUS FIBRIN \\ ADHESIVE
}

\author{
MIRANDA-ROLLÓN MD ${ }^{1}$, PÉREZ-GONZÁLEZ LE ${ }^{1}$, SENTIERI-OMARREMENTERÍA A ${ }^{1}$, \\ MARTÍNEZ-RODRÍGUEZ $\mathrm{R}^{1}$, PARENTE-HERNÁNDEZ $\mathrm{B}^{1}$, JUNCEDA-MORENO J ${ }^{2}$
}

\section{RESUMEN}

Objetivo: Comparar la tasa de recurrencias, complicaciones y hallazgos biomicroscópicos después de cirugía de pterigión con autoinjerto conjuntival usando adhesivo tisular (Tissucol Duo ${ }^{\circledR}$, Baxter AG, Viena, Austria) o sutura (seda 7-0).

Material y métodos: Se han incluido en el estudio pacientes con pterigión nasal en dos grupos de cirugía de autoinjerto conjuntival: en 9 de ellos el injerto se sutura a conjuntiva y en 8 de ellos se fija con adhesivo tisular.

Resultados: Se intervinieron 17 ojos de 17 pacientes, $41,2 \%$ mujeres y $58,8 \%$ hombres con edad media de 59,8 años. 5 ojos presentaban recurrencia de resección simple y 12 de ellos presentaban pterigión primario. El tamaño de la invasión corneal osciló entre 2 y $4 \mathrm{~mm} .33,3 \%$ de los pacientes del grupo de sutura presentaron molestias después de la cirugía frente a ninguno en el grupo de adhesivo. $44,4 \%$ de los pacientes presentaron inflamación tras la cirugía frente a ninguno en el grupo de sutura. En uno de los pacientes del grupo del adhesivo se pro-

\begin{abstract}
Purpose: To compare recurrence rate, complications, and biomicroscopical findings after conjunctival autograft pterygium surgery with the use of a fibrin glue (Tissucol Duo ${ }^{\circledR}$, Baxter AG, Vienna, Austria) or suture (7-0 silk).

Methods: Patients with nasal pterygium were included in two groups of conjunctival autograft surgery. In 9 of them the graft was sutured to the surrounding conjunctiva and in 8 of them the graft was fixed to the conjunctiva using fibrin glue.

Results: 17 patients (17 eyes) $41.2 \%$ women and $58.8 \%$ men were surgically treated. Mean patient age was 59.8 years. Five of the patients presented recurrence for simple excision and 12 presented primary pterygium. The extent of corneal invasion was 2 to $4 \mathrm{~mm}$. In the suture group, $33.3 \%$ of the patients experienced pain after surgery compared to none in the fibrin glue group. In the suture group, $44.4 \%$ of the patients presented inflammation after surgery compared to none in the glue group. One patient from the glue group lost the graft and presented a recurrence one month later.
\end{abstract}

\footnotetext{
Recibido: 31/5/08. Aceptado: 1/4/09.

Servicio de Oftalmología. Hospital de Cabueñes. Gijón. España.

${ }^{1}$ Licenciado en Medicina.

2 Doctor en Medicina.

Correspondencia:

María Dolores Miranda Rollón

C/. Teodoro Cuesta, $13,6 .^{\circ} \mathrm{B}$

33012 Oviedo (Asturias)

España

E-mail: mdmr1980@ hotmail.com
} 
dujo una pérdida del injerto causando una posterior recidiva del pterigión.

Conclusiones: El uso de adhesivo tisular reduce los síntomas postquirúrgicos así como la inflamación y el disconfort. La tasa de recurrencias parece ser similar con los dos procedimientos.

Palabras clave: Cirugía de pterigión, autoinjerto conjuntival, adhesivo tisular, recurrencias, disconfort postquirúrgico.
Conclusions: The use of fibrin glue in pterygium surgery reduces patient symptoms, inflammation and discomfort. The rate of recurrence seems to be similar in both procedures (Arch Soc Esp Oftalmol 2009; 84: 179-184).

Key words: Pterygium surgery, autograft, fibrin glue, recurrence, postoperative discomfort.

\section{INTRODUCCIÓN}

El término pterigión deriva del término griego pterygion que significa «aleta». Se trata de un sobrecrecimiento de tejido conectivo fibrovascular de la conjuntiva sobre la córnea que puede llegar a afectar a la visión (1).

El principal problema de esta entidad es que a pesar de que a lo largo de la historia de la Oftalmología se han empleado diferentes técnicas para su tratamiento la tasa de recurrencias todavía sigue siendo elevada.

La cirugía del pterigión está sometida a continuas modificaciones que buscan el modo de disminuir la tasa de recidivas de la misma.

La técnica de la resección con autoinjerto conjuntival ha demostrado buenos resultados en este aspecto debido a que mantiene regular la superficie ocular y restaura la anatomía previa a la invasión corneal producida por el pterigión anclándose al lecho escleral desnudo mediante suturas como puede ser la seda o la poliglactina o mediante los adhesivos titulares (2).

Ambas técnicas aportan excelentes resultados a la hora de la disminución del número de recurrencias en este tipo de cirugía. Además otro factor importante en este tipo de cirugía es el confort del paciente los días posteriores a la intervención.

Basándonos en esto se realizan las dos técnicas con pacientes afectados de pterigión en nuestro hospital tratando de evaluar los siguientes datos:

1. Complicaciones quirúrgicas y postquirúrgicas de las dos técnicas.

2. Hallazgos biomicroscópicos postquirúrgicos de los dos procedimientos.

3. Molestias subjetivas de los pacientes en ambos grupos tras la cirugía.

4. Determinar si existen diferencias en cuanto al número de recidivas.

\section{SUJETOS, MATERIAL Y MÉTODO}

Se trata de un estudio observacional retrospectivo de los pacientes intervenidos de cirugía de pterigión con autoinjerto en nuestro centro por dos cirujanos desde enero 2006 hasta enero 2007.

Se incluyeron en el estudio pacientes con invasión corneal del pterigión mayor de $2 \mathrm{~mm}$, que fuese sintomático o recidiva tras una resección simple previa.

Se excluyeron pacientes con recurrencia de pterigión de técnica de autoinjerto o pacientes en los que se preveía la necesidad de realización de cirugía filtrante posterior en casos de glaucoma.

Para la clasificación del pterigión se usa el sistema del grupo de Madrid de acuerdo a: atrófico (vascularización por debajo del cuerpo del pterigión), carnoso (vascularización en el cuerpo del pterigión) e intermedio.

Los pacientes, tras firmar consentimiento informado, se incluyen en dos grupos según el tratamiento quirúrgico a relizar: 9 pacientes son tratados con la técnica de autoinjerto conjuntival con sutura del mismo y los otros 8 con la misma técnica de autoinjerto pero con adhesivo tisular (Tissucol Duo $^{\circledR}$, Baxter AG, Vienna, Austria).

\section{Técnica quirúrgica}

Excepto en uno de los pacientes que se preveía una mala colaboración y se optó por la anestesia peribulbar en el resto de los pacientes se empleó anestesia tópica (colirio de tetracaína) junto con anestesia subconjuntival (bupivacaína con vasoconstrictor) aplicado debajo del pterigión y en la zona conjuntival donante.

Posteriormente se realiza queratectomía con cuchillete de $45^{\circ}$ intentado separar bien el tejido 
del pterigión adherido a la córnea procurando no dejar restos ni escalones. A continuación se extirpa la cabeza del pterigión y la cápsula de Tenon subyacente con tijera de Westcott intentando dejar el lecho escleral limpio de cápsula de Tenon al igual que los márgenes de conjuntiva perilesional prestando especial cuidado a la zona próxima a carúncula para no lesionar las fibras de inserción del recto medio. En casos de vascularización escleral densa secundaria a la intensa inflamación se aplicó cauterización a baja intensidad con diatermia bipolar sobre la zona.

El autoinjerto se toma de la conjuntiva superior del mismo ojo midiendo con regla el lecho escleral y llevando estas medidas a esta zona pidiendo al paciente que mire hacia abajo para una adecuada exposición de la zona (desde el borde limbar hasta el fondo de saco) y marcando los bordes con un rotulador quirúrgico.

$\mathrm{Al}$ infiltrar la anestesia sobre esta zona se procura hacer hidrodisección separando la conjuntiva de la cápsula de Tenon. Se diseca la zona con tijera de Westcott empezando por el fondo de saco dirigiéndonos hacia limbo. Una vez obtenido el injerto se corta por la zona limbar y se voltea sobre la córnea con mucho cuidado. A continuación se gira el injerto sobre la córnea hasta enfrentarlo al lecho escleral manteniendo la orientación yuxtalimbarlimbo.

En la variante con sutura se ancla el injerto a conjuntiva perilesional con seda 7-0 a modo de puntos sueltos.

En la variante de adhesivo se coloca una gota de trombina en el lecho escleral y una de fibrinógeno en la cara estromal del injerto juntando ambas partes y presionando unos 10 segundos sobre el injerto con una hemosteta.

Tras la operación en ambas técnicas se hizo cura tópica con ciclopléjico, combinado de tobramicinadexamentasona y pomada de gentamicina con oclusión semicompresiva.

Los pacientes fueron revisados al día siguiente retirando el vendaje y el ciclopléjico, manteniendo la pomada y la combinación de corticoide y antibiótico el primer mes. Se hacen revisiones a los 15 días, momento en que se retira la sutura en este grupo de pacientes, a los dos meses y a los seis meses realizando examen oftalmológico de agudeza visual, biomicroscopía y toma de presión intraocular (figs. 1 y 2 ).

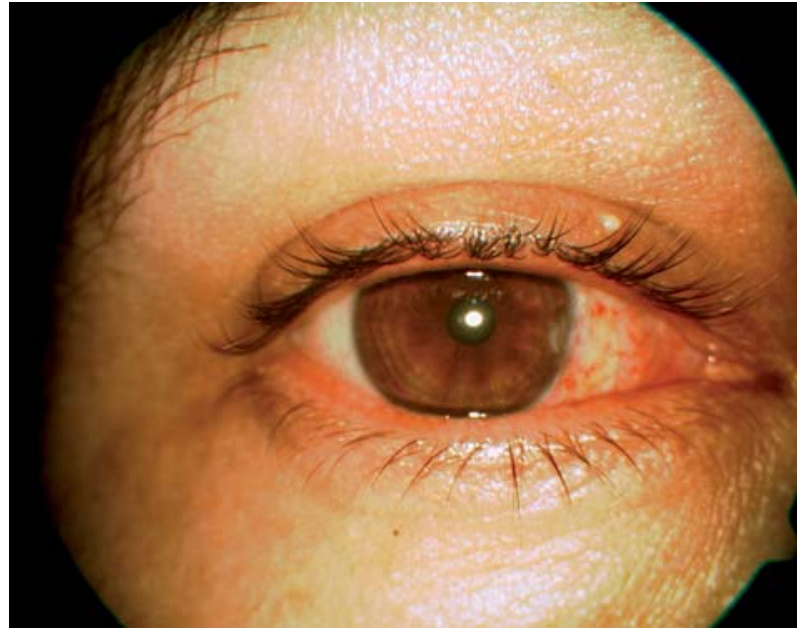

Fig. 1: Aspecto postquirúrgico de autoinjerto con adhesivo tisular.

\section{Análisis estadístico}

Las variables analizadas fueron edad, sexo, antecedentes personales de riesgo (traumatismos oculares, trabajo en el campo, procedencia de país con tasa solar elevada), existencia de cirugía previa, tipo de pterigión, milímetros de invasión corneal, síntomas y signos postquirúrgicos y la presencia o no de recidivas.

Para las variables cuantitativas se comprueba si siguen una distribución normal dentro de cada grupo de cirugía mediante el test de Shapiro-Wilk. Así para comparar la edad media y los milímetros de invasión corneal según la cirugía se utilizó la prueba de la t de Student.

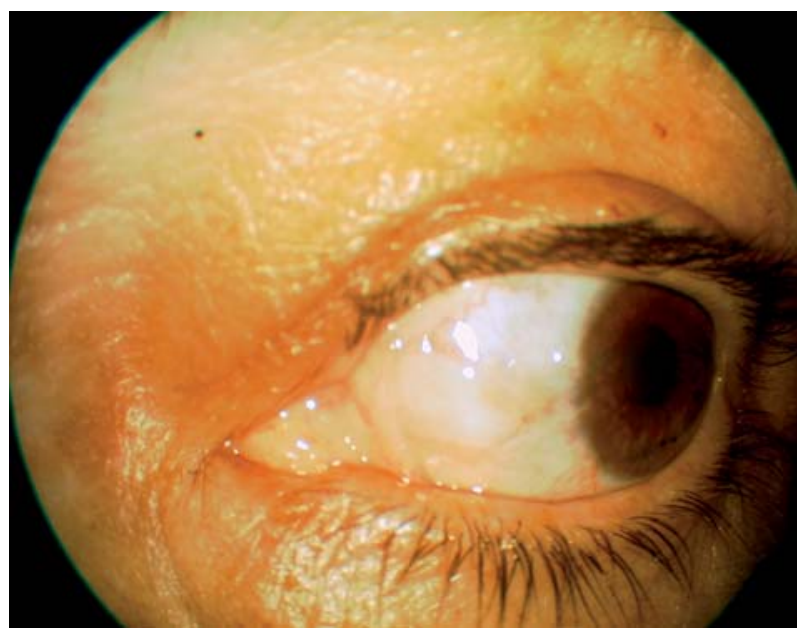

Fig. 2: Aspecto postquirúrgico de autoinjerto con sutura. 
Para las variables cualitativas se utilizan tablas de contingencia para analizar la relación entre el tipo de cirugía y dichas variables. Posteriormente se contrasta la hipótesis de independencia con el test exacto de Fisher para tablas de 2x2 el cual tiene la principal ventaja de poder ser usado para muestras de tamaño muy pequeño como es ésta.

En todos los casos se considera como estadísticamente significativo la existencia de una probabilidad de error inferior al 5\% $(\mathrm{p}<0,05)$.

\section{RESULTADOS}

Se recogieron 17 ojos operados de pterigión (tabla I), de los cuales siete eran mujeres $(41,2 \%)$ y diez eran hombres $(58,8 \%)$ con una edad media de 59,8 años (rango de 36 a 81 años). La edad media en el grupo de sutura fue de 52 años y en el grupo de adhesivo 62 años.

Cinco pacientes del total ya habían sido operados de pterigión mediante resección simple, tres en el grupo intervenido mediante sutura y 2 en el grupo intervenido con adhesivo tisular $(\mathrm{p}=1,00)$.

El tamaño de invasión corneal prequirúrgico del pterigión osciló entre 2 y 4 mm no hallándose tampoco diferencias significativas en el tamaño medio del pterigión entre los dos grupos $(\mathrm{p}=0,097)$.

Tres pacientes en el grupo de ojos intervenidos con sutura $(33,3 \%)$ refirieron molestias en el postoperatorio frente a ninguno en el grupo de tissucol, no siendo este resultado, sin embargo, estadísticamente significativo $(\mathrm{p}=0,12)$.

En cuanto a los signos postquirúrgicos cuatro de los pacientes en el grupo de sutura $(44,4 \%)$ presentaron inflamación intensa con quémosis conjuntival en el primer día del postoperatorio, presentando uno de ellos un granuloma conjuntival. Dos de los pacientes del grupo de adhesivo presentaron despegamiento del injerto. En ambos casos se dejó cicatrizar por segunda intención. Estos resultados no resultaron estadísticamente significativos en el análisis estadístico $(\mathrm{p}=0,58)$.

El número de recidivas fue de tan sólo una en el grupo de adhesivo (fig. 3) coincidiendo con uno de los pacientes en los que se perdió el injerto por el despegamiento del mismo tras la cirugía.

\section{DISCUSIÓN}

Son numerosas las técnicas empleadas en la cirugía del pterigión desde las más sencillas como son la avulsión o la resección simple hasta otras más complejas como puede ser la queratoplastia lamelar (3), pasando por los recubrimientos con diferentes tejidos como puede ser la membrana amniótica y el uso de antimetabolitos coadyuvantes como mitomicina y daunorrubicina (4).

Todas ellas tienen tres objetivos principales: disminuir la tasa de recidivas, evitar las complicaciones quirúrgicas y postquirúrgicas y obtener un

Tabla I. Tabla de recogida de datos de los pacientes intervenidos de pterigión

\begin{tabular}{|c|c|c|c|c|c|c|c|c|c|c|}
\hline Paciente & Edad & Sexo & Ojo & Cir. previas & Tipo & Tamaño & Cirugía & Síntomas & Signos & Recidiva \\
\hline 1 & 55 & $\mathrm{~m}$ & d & no & carnoso & 4 & sutura & no & no & no \\
\hline 2 & 46 & $\mathrm{~h}$ & $\mathrm{~d}$ & sí & carnoso & 3 & sutura & no & hiperemia & no \\
\hline 3 & 53 & $\mathrm{~h}$ & $\mathrm{i}$ & no & carnoso & 2 & sutura & sí & no & no \\
\hline 4 & 64 & $\mathrm{~m}$ & $\mathrm{i}$ & no & intermedio & 2 & sutura & sí & hiperemia & no \\
\hline 5 & 36 & $\mathrm{~h}$ & $\mathrm{~d}$ & no & carnoso & 3 & sutura & no & no & no \\
\hline 6 & 73 & $\mathrm{~h}$ & $\mathrm{~d}$ & no & atrófico & 2 & sutura & sí & no & no \\
\hline 7 & 70 & $\mathrm{~m}$ & $\mathrm{~d}$ & sí & carnoso & 2,5 & sutura & no & no & no \\
\hline 8 & 62 & $\mathrm{~h}$ & $\mathrm{~d}$ & sí & carnoso & 4 & sutura & no & granuloma & no \\
\hline 9 & 60 & $\mathrm{~m}$ & $\mathrm{i}$ & no & carnoso & 4 & sutura & no & hiperemia & no \\
\hline 10 & 53 & $\mathrm{~h}$ & $\mathrm{~d}$ & no & carnoso & 2,5 & tisucol & no & no & no \\
\hline 11 & 82 & $\mathrm{~m}$ & $\mathrm{~d}$ & no & atrófico & 3 & tisucol & no & no & no \\
\hline 12 & 70 & $\mathrm{~h}$ & $\mathrm{i}$ & no & carnoso & 3 & tisucol & no & no & no \\
\hline 13 & 36 & $\mathrm{~m}$ & $\mathrm{i}$ & no & carnoso & 4 & tisucol & no & no & no \\
\hline 14 & 51 & $\mathrm{~m}$ & $\mathrm{~d}$ & no & intermedio & 2 & tisucol & no & no & no \\
\hline 15 & 63 & $\mathrm{~h}$ & $\mathrm{i}$ & sí & atrófico & 3 & tisucol & no & despegamiento & no \\
\hline 16 & 81 & $\mathrm{~h}$ & $\mathrm{~d}$ & sí & carnoso & 4 & tisucol & no & despegamiento & Sí \\
\hline 17 & 62 & $\mathrm{~h}$ & $\mathrm{~d}$ & no & atrófico & 3,5 & tisucol & no & no & no \\
\hline
\end{tabular}

$\mathrm{m}=$ mujer; $\mathrm{h}=$ hombre; $\mathrm{d}=$ derecho; $\mathrm{i}=$ izquierdo; cir. previas= cirugías previas; tamaño= tamaño de invasión corneal medido en milímetros. 


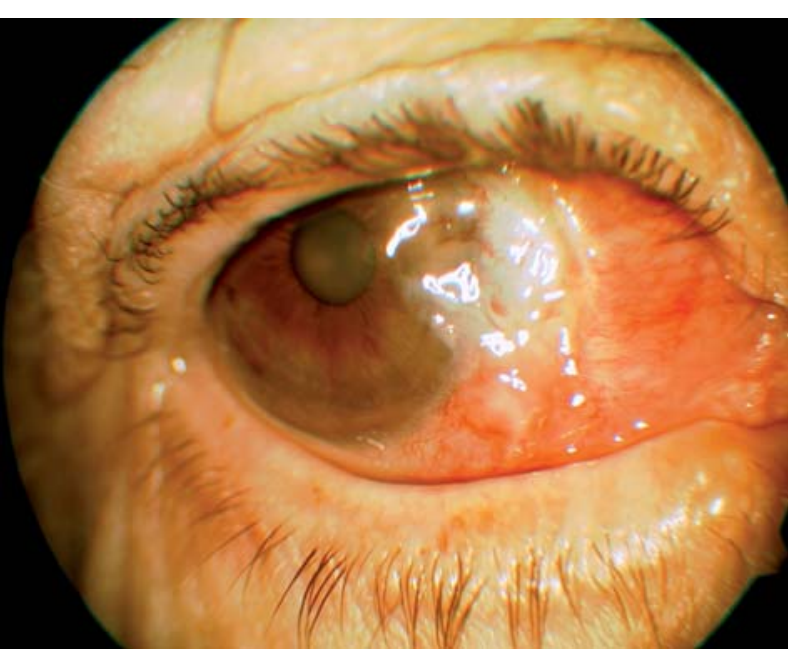

Fig. 3: Pterigión recidivado de autoinjerto con adhesivo tisular.

resultado cosmético satisfactorio (5). Para ello es necesario causar una mínima inflamación y tratar de inhibir la angiogénesis procurando, mediante la cirugía, dejar el lecho escleral lo más limpio posible, frenar el crecimiento del tejido subconjuntival con medicación antiinflamatoria y tratar de restaurar la función de la barrera limbar (6).

La tasa de recurrencia del pterigión en las distintas series publicadas va desde 2 a $39 \%$ después de la cirugía siendo las recidivas más frecuentes y rápidas las producidas tras cirugía de resección simple (7). Por el contrario la técnica quirúrgica que mejores resultados ha conseguido a la hora de disminuir el número de recidivas es la técnica del autoinjerto conjuntival (8).

Hoy en día son muchos los objetivos que se buscan tras la cirugía del pterigión no quedándonos sólo en lograr la no recidiva a largo plazo sino también conseguir disminuir el tiempo de la cirugía, lograr el mayor confort postquirúrgico del paciente tras la cirugía y evitar el dolor en los días posteriores. En nuestro estudio el 44,4\% de los pacientes del grupo de sutura presentaron quémosis e inflamación importante en el postoperatorio frente al $0 \%$ en el grupo de tissucol y el 33,3\% de los pacientes manifestaron molestias en el grupo intervenido con sutura frente ninguno de losque fueron intervenidos con adhesivo tisular. Estos resultados a pesar de no ser estadísticamente significativos, probablemente por el bajo tamaño muestral, coinciden con los publicados en otras series de mayor tamaño (9-11) en las que se encuentran mejores resultados cosmé- ticos y en cuanto a confort en los primeros días postquirúrgicos en los grupos intervenidos mediante autoplastia con adhesivo tisular. Se ha postulado que esto puede ser debido también a una cierta capacidad antiinflamatoria de los adhesivos tisulares (10) frente a una actividad proinflamatoria de las suturas que puede incluso llevar a la formación de granulomas (12).

La principal complicación acaecida en el postoperatorio, fue la pérdida del injerto en dos de los pacientes intervenidos mediante adhesivo tisular. Esta complicación ha sido descrita en otra serie en un paciente intervenido mediante la misma técnica (9).

En cuanto al número de recidivas, en nuestro estudio hemos presentado una en el grupo intervenido con adhesivo tisular, debida a la pérdida del injerto, no existiendo ninguna en el grupo intervenido con sutura. En los estudios al respecto sólo se encontraron diferencias entre ambos tipos de técnicas en un estudio, publicado por Korangi y cols $(13,14)$ los cuales encontraron un mayor número de recidivas en pacientes intervenidos con sutura.

En conclusión, el uso del adhesivo causa un confort mayor en el postoperatorio ya que con la reducción del tiempo de la cirugía y sus propiedades antiinflamatorias provoca menos síntomas postquirúrgicos que la sutura. Sin embargo existe la posibilidad de pérdida del injerto tisular en los días posteriores debida al despegamiento de éste con las consiguientes complicaciones que ello puede conllevar y que en este caso supuso la recidiva del pterigión.

\section{BIBLIOGRAFÍA}

1. Iradier Urrutia MT. Cirugía del pterigión. Madrid: Macline. 2006.

2. Barraquer RI. Pterigión y pinguécula. En: Benítez del Castillo JM, Durán de la Colina JA, Rodríguez Ares MT. Superficie Ocular. Madrid: Sociedad Española de Oftalmología. 2004.

3. Sangwan V, Murthy SI, Bansal AK. Surgical treatment of chronically recurring pterygium. Cornea 2003; 22: 63-65.

4. Dadeya S. Autografting of marginal conjuntiva. Ophtalmologica 2003; 217: 237-238.

5. Maheshwari S. Split- conjuntival grafts for double head pterygium. Indian J Opth 2005; 53: 53-55.

6. Dadeya S, Malik KP, Gulliani BP. Pterygium surgery: conjuntival rotation autograft versus conjuntival autograft. Ophtalmic Surg Lasers 2002; 33: 269-274.

7. Karabatsas CH, Marsh GW, Cook AM. Different therapeutic approaches and outcome in the treatment of pterygium. Eur J Ophtalmol 1998; 8: 148-152. 
8. Varssano D, Michaeli-Cohen A, Loewenstein A. Excision of pterygium and conjuntival autograft. Isr Med Assoc J 2002; 4: 1097-1100.

9. Uy HS, Reyes JM, Flores JD. Comparison fibrin glue and sutures for attaching conjuntival autografts after pterigium excision. Ophtalmology 2005; 112: 667-671.

10. Bahar I, Weinberger D, Dan G. Pterygium surgery: fibrin glue versus vicryl sutures for conjuntival closure. Cornea 2006; 25: 1168-1172.

11. Marticorena J, Rodriguez Ares MT, Touriño R. Pterygium surgery: conjuntival autograft using a fibrin adhesive. Cornea 2006; 25: 34-36.

12. Assia E. Surgical management of pterygium. Isr Med Assoc J 2002; 4: 1138-1139.

13. Korangy $G$, Seregard S, Kopp ED. Cut and paste: a no suture, small incision approach to pterygium surgery. Br J Ophthalmol 2004; 88: 911-914.

14. Korangy $G$, Seregard S, Kopp ED. The cut and paste method for primary surgery: long- term follow- up. Acta Opthalmol Scand 2005; 83: 298-301. 